\title{
Flexible diel vertical migration behaviour of zooplankton in the Irish Sea
}

\author{
Xabier Irigoien ${ }^{1, *}$, David V. P. Conway ${ }^{2}$, Roger P. Harris ${ }^{3}$ \\ ${ }^{1}$ AZTI, Herrera Kaia Portualdea, Pasaia 20110, Guipúzcoa, Spain \\ ${ }^{2}$ Marine Biological Association, The Laboratory, Citadel Hill, Plymouth PL1 2PB, UK \\ ${ }^{3}$ Plymouth Marine Laboratory, Prospect Place, Plymouth PL1 3DH, UK
}

\begin{abstract}
The diel vertical migration (DVM) of the whole plankton community was investigated in the central and coastal Irish Sea. Generally, more than $60 \%$ of the plankton did not perform significant DVM. A correlation analysis of the weighted mean depths of different organisms and their potential predators suggested relationships between 2 groups: Oithona spp., copepod nauplii and fish larvae, and Calanus spp., Pseudocalanus elongatus and chaetognaths (Sagitta spp.). Organisms showing significant DVM were chaetognaths (Sagitta spp.), Calanus spp. and P. elongatus. Calanus spp. showed clear ontogenic variations in DVM and, along with $P$. elongatus, demonstrated great flexibility in both the amplitude and direction of migration. $P$. elongatus did not migrate in the coastal area and Calanus spp. showed a clear reverse migration. The direction of migration appeared to be related to the vertical position of the chaetognaths in the water column during the day.
\end{abstract}

KEY WORDS: Diel vertical migration · Calanus spp. · Pseudocalanus spp. · Oithona spp. C Chaetognaths · Fish larvae Resale or republication not permitted without written consent of the publisher

\section{INTRODUCTION}

Changes in species composition are the consequence of species-specific behavioural changes, as differences in behaviour result in differences in the main demographic parameters, birth and mortality (Ohman 1990), and consequently in the success or failure of the species in a changing ecosystem. For example, a very well adapted but rigid strategy to avoid one specific predator can become a disadvantage if the predator is replaced as the main cause of mortality in the system. One particular predation avoidance behaviour is characteristic of plankton, diel vertical migration (DVM) (Lampert 1993). While it is now generally accepted that DVM is a predation avoidance mechanism (Lampert 1993), there are contradictory cases, such as species that do not appear to migrate (Hays et al. 1997) or lack of relation with predator abundance (Huntley \& Brooks 1982, Dale \& Kaartvedt 2000). However, this type of fixed DVM behaviour (not related to changes in predators) has been attributed to relatively constant mortality risks (Neill 1992).
Studies on DVM behaviour often only include 1 or 2 species of migrating organisms (e.g. Ohman 1990, Hays et al. 1995), and when there is information on a larger number of species these are generally copepods and/or euphausiids (Moraitou-Apostolopoulou 1971, Andersen et al. 2001). Although some authors have described day/night changes in vertical distribution for a range of components (e.g. Russell 1930, Roe 1974, Longhurst 1985, Ashjian et al. 1998), the number of taxonomically detailed studies investigating to what extent DVM is a generally adopted strategy in marine planktonic communities is limited. In this study, we examined the DVM of the whole mesozooplankton and macrozooplankton community (including meroplankton). We had 2 objectives: (1) to determine whether DVM is a general behaviour among planktonic organisms, and (2) to determine how flexible a behaviour DVM is, both in terms of differences between groups and species and in terms of differences within the same species under different conditions. 


\section{MATERIALS AND METHODS}

Sampling was carried out during a series of cruises between April 1987 and April 1989 (Table 1) at 2 hydrographically contrasting sites in the Irish Sea: (1) a site of $\sim 40 \mathrm{~m}$ depth close to the Irish coast with a weak seasonal thermal stratification, and (2) a more central Irish Sea site of $\sim 120$ m depth which becomes more strongly seasonally stratified during the summer. We took 2 zooplankton hauls at each site, on 1 night and 1 day (around midday and midnight), using a Longhurst Hardy Plankton Recorder (LHPR; Williams et al. 1983). The LHPR is a high-speed multiple serial sampler, and was fitted with a coarse (200 $\mu \mathrm{m})$-mesh net collecting system. Zooplankton are collected between 2 rolls of filtering gauze, which are advanced at intervals inside a cod-end box to give a series of consecutive samples.

The LHPR was towed at 3 to 4 knots on long oblique hauls, sampling from the surface to around $5 \mathrm{~m}$ off the bottom (see Table 1) at 1 min intervals, with individual sample-depth horizons of approximately $5 \mathrm{~m}$, filtering approximately $10 \mathrm{~m}^{3}$ of water for each sample. The number of samples taken during each haul varied between 9 and 33 (Table 1), so that the distance covered during the hauls was around 0.5 to $2.0 \mathrm{n}$ miles. A temperature probe on the control unit recorded the water temperature associated with each sample, and a flowmeter in the mouth of the net recorded the volume filtered. The individual samples were washed off the filtering gauzes and preserved in $4 \%$ borax-buffered formaldehyde, prepared with freshwater. Large organisms (euphausiids, de-

Table 1. Plankton sampling details. N: no. of samples

\begin{tabular}{|lcccrc|}
\hline $\begin{array}{l}\text { Date } \\
\text { (d/mo/yr) }\end{array}$ & Time & Position & $\begin{array}{r}\text { Bottom } \\
\text { depth }(\mathrm{m})\end{array}$ & $\begin{array}{r}\text { Sampling } \\
\text { depth }(\mathrm{m})\end{array}$ & $\mathrm{N}$ \\
\hline $\begin{array}{c}\text { Coastal area } \\
\text { 09/04/87 }\end{array}$ & $12: 38$ & $53^{\circ} 53^{\prime} \mathrm{N}, 05^{\circ} 58^{\prime} \mathrm{W}$ & 41 & 35 & 13 \\
$09 / 04 / 87$ & $23: 05$ & $53^{\circ} 53^{\prime} \mathrm{N}, 05^{\circ} 57^{\prime} \mathrm{W}$ & 41 & 39 & 13 \\
$11 / 04 / 88$ & $23: 29$ & $53^{\circ} 47^{\prime} \mathrm{N}, 05^{\circ} 49^{\prime} \mathrm{W}$ & 63 & 62 & 14 \\
$12 / 04 / 88$ & $11: 56$ & $53^{\circ} 45^{\prime} \mathrm{N}, 05^{\circ} 50^{\prime} \mathrm{W}$ & 56 & 50 & 12 \\
$21 / 04 / 89$ & $12: 29$ & $53^{\circ} 50^{\prime} \mathrm{N}, 06^{\circ} 06^{\prime} \mathrm{W}$ & 31 & 28 & 9 \\
$21 / 04 / 89$ & $23: 01$ & $53^{\circ} 48^{\prime} \mathrm{N}, 06^{\circ} 05^{\prime} \mathrm{W}$ & 31 & 28 & 11 \\
Central Irish Sea & & & & & \\
$19 / 02 / 87$ & $01: 45$ & $53^{\circ} 50^{\prime} \mathrm{N}, 05^{\circ} 32^{\prime} \mathrm{W}$ & 96 & 92 & 26 \\
$19 / 02 / 87$ & $10: 14$ & $53^{\circ} 50^{\prime} \mathrm{N}, 05^{\circ} 32^{\prime} \mathrm{W}$ & 97 & 95 & 26 \\
$11 / 04 / 87$ & $12: 10$ & $53^{\circ} 50^{\prime} \mathrm{N}, 05^{\circ} 28^{\prime} \mathrm{W}$ & 130 & 115 & 33 \\
$11 / 04 / 87$ & $23: 46$ & $53^{\circ} 50^{\prime} \mathrm{N}, 05^{\circ} 28^{\prime} \mathrm{W}$ & 130 & 120 & 32 \\
$14 / 04 / 88$ & $12: 09$ & $53^{\circ} 51^{\prime} \mathrm{N}, 05^{\circ} 32^{\prime} \mathrm{W}$ & 102 & 100 & 27 \\
$15 / 04 / 88$ & $23: 23$ & $53^{\circ} 51^{\prime} \mathrm{N}, 05^{\circ} 32^{\prime} \mathrm{W}$ & 108 & 106 & 24 \\
$25 / 05 / 88$ & $00: 13$ & $53^{\circ} 49^{\prime} \mathrm{N}, 05^{\circ} 33^{\prime} \mathrm{W}$ & 93 & 85 & 24 \\
$25 / 05 / 88$ & $12: 47$ & $53^{\circ} 49^{\prime} \mathrm{N}, 05^{\circ} 31^{\prime} \mathrm{W}$ & 105 & 100 & 20 \\
$22 / 04 / 89$ & $23: 09$ & $53^{\circ} 45^{\prime} \mathrm{N}, 05^{\circ} 29^{\prime} \mathrm{W}$ & 104 & 100 & 25 \\
$23 / 04 / 89$ & $12: 42$ & $53^{\circ} 45^{\prime} \mathrm{N}, 05^{\circ} 30^{\prime} \mathrm{W}$ & 107 & 99 & 22 \\
& & & & & \\
\hline
\end{tabular}

capods, coelenterates, chaetognaths, fish larvae and fish eggs) were counted using the whole sample, while species analysis and counts for smaller organisms such as copepods and meroplankton were carried out on subsamples. Conductivity, temperature, depth (CTD) profiles were obtained either by a Neil Brown or a Guildline CTD rosette array, and were taken before or after each LHPR haul, to provide additional environmental information. Weighted mean depth (WMD) of each taxonomic group was calculated as:

$$
\left.\mathrm{WMD}=\left(\sum n_{\mathrm{i}} d_{\mathrm{i}}\right) / \sum n_{\mathrm{i}}\right)
$$

where $n_{\mathrm{i}}$ is the abundance of organisms at depth $d_{\mathrm{i}}$ (Worthington 1931). The amplitude of migration was calculated as the difference between the WMD between day and night. A positive value indicated movement towards the surface during the night and a negative value reverse vertical migration.

All sampling strategies have problems, and ours had 3 main limitations: (1) Since the LHPR does not sample $5 \mathrm{~m}$ above the bottom, the weighted mean depth would be underestimated for species that accumulate near the bottom; this could particularly be the case for Sagitta spp. that have been observed at high concentrations near the bottom, especially during the daytime (Pearre 1973, Choe \& Deibel 2000). (2) The accuracy of the amplitude estimation is related to the abundance of organisms, and therefore estimations for organisms present in low abundance must be treated with caution. Consequently, our data on migration amplitude concentrate on the most abundant organisms and their potential predators. Accordingly, different species of fish larvae were grouped to obtain a more accurate estimation of the DVM (details of the diurnal and nocturnal vertical distribution of larval fish species are given in Conway et al. 1997). (3) The migration patterns of many migrators differ at different times during dark and light. Thus, a single set of night and day samples represented gives but a general indication of DVM. While it can detect many instances of DVM, it often underestimates ranges and sometimes fails to detect migration at all (Pearre 2000). Therefore, the ranges presented here must be viewed with caution.

\section{RESULTS}

\section{Temperature and stratification}

In the coastal area, sea surface temperature (SST) ranged from $6.7^{\circ} \mathrm{C}$ on 09 April 1987 to $8.34^{\circ} \mathrm{C}$ on 21 April 
1989. As expected for this strongly tidally mixed area, there was no stratification of the water column (Fig. 1). In the central Irish Sea, SST during the April cruises ranged from $7^{\circ} \mathrm{C}$ on 11 April 1987 to $8.8^{\circ} \mathrm{C}$ on 23 April 1989 , with a maximum of $10.5^{\circ} \mathrm{C}$ on 25 May 1988. During the April cruises there was only weak stratification, but a clearly defined thermocline appeared at about 30 m during May 1988 (Fig. 1).

\section{Community composition}

The total abundance of zooplankton (ind. $\mathrm{m}^{-2}$ ) was generally 2 to 3 times higher in the central Irish Sea than in the coastal area, however, taking the depth difference into consideration, there were no systematic differences in density (ind. $\mathrm{m}^{-3}$ ) (Table 2). During the spring, Paracalanus parvus, Pseudocalanus elongatus, Oithona spp. and meroplanktonic groups, such as cirripede nauplii, echinoderm larvae, decapod larvae and occasionally the cyphonautes larvae of bryozoans, generally dominated the population. During the summer cruise in 1988, Acartia spp. were also an important component of the population. The meroplankton contribution to the total population was generally no lower in the central Irish Sea than in the coastal area. During 1989 there seemed to be a shift in numerical dominance from Paracalanus parvus to Pseudocalanus elongatus and from Calanus finmarchicus to C. helgolandicus.

\section{Diel vertical migration}

There was a high variability in DVM, both between and within groups or species (Figs. 2 \& 3, Table 3). In general, mean depth of 60 to $80 \%$ of the organisms did not vary by more than $10 \mathrm{~m}$ (2 sample-depth hori-
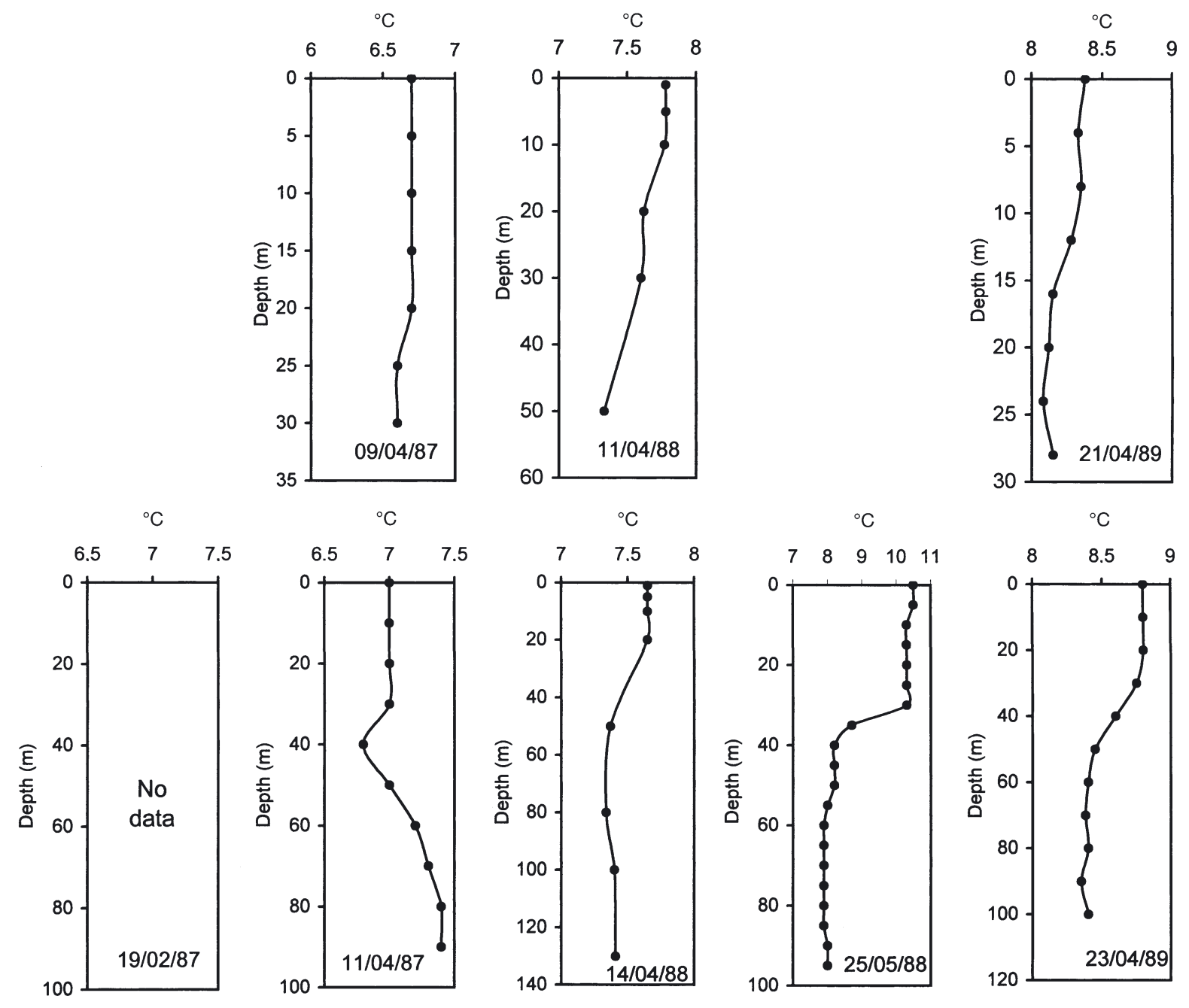

Fig. 1. Temperature profiles of stations sampled in the coastal (upper panels) and central (lower panels) Irish Sea. Dates are given as $\mathrm{d} / \mathrm{mo} / \mathrm{yr}$ 
Table 2. Abundance (ind. $\mathrm{m}^{-2}$ ) of zooplankton species/groups sampled at coastal and central Irish Sea stations. Dates are given as d/mo/yr

\begin{tabular}{|c|c|c|c|c|c|c|c|c|c|c|c|c|c|c|c|c|}
\hline & \multicolumn{6}{|c|}{${ }$ Coastal $\longrightarrow$} & \multirow{2}{*}{\multicolumn{2}{|c|}{$12 / 02 / 87$}} & \multirow{2}{*}{\multicolumn{2}{|c|}{$11 / 04 / 87$}} & \multirow{2}{*}{\multicolumn{2}{|c|}{$\begin{array}{l}\text { - Central- } \\
14 / 04 / 88\end{array}$}} & \multirow{2}{*}{\multicolumn{2}{|c|}{$25 / 05 / 88$}} & \multirow{2}{*}{\multicolumn{2}{|c|}{$22 / 04 / 89$}} \\
\hline & \multicolumn{2}{|c|}{$09 / 04 / 87$} & \multicolumn{2}{|c|}{$11 / 04 / 88$} & \multicolumn{2}{|c|}{$21 / 04 / 89$} & & & & & & & & & & \\
\hline & Day & Night & Day & Night & Day & Night & Day & Night & Day & Night & Day & Night & Day I & Night & Day & Night \\
\hline Acartia spp. I-IV & 325 & 1087 & 415 & 467 & 302 & 292 & 20 & 13 & 540 & 1277 & 2338 & 2410 & 42922 & 115211 & 30 & 98 \\
\hline Acartia spp. V & 123 & 326 & 397 & 382 & 239 & 189 & - & 20 & 309 & 619 & 926 & 1316 & 24713 & 14223 & - & 25 \\
\hline Acartia spp. VI & 161 & 279 & 1117 & 965 & 821 & 1596 & 11 & 64 & 226 & 547 & 2809 & 3434 & 18996 & 35419 & 134 & 122 \\
\hline Calanus spp. CI & 183 & 331 & 315 & 268 & 46 & 731 & - & - & 456 & 223 & 1789 & 1621 & 9810 & 1188 & 241 & 96 \\
\hline Calanus spp. CII & 234 & 413 & 667 & 543 & 142 & 245 & - & - & 420 & 440 & 1022 & 1485 & 6918 & 1737 & 133 & 261 \\
\hline Calanus spp. CIII & 111 & 126 & 629 & 122 & 387 & 367 & 3 & - & 364 & 682 & 408 & 607 & 7686 & 7363 & 204 & 212 \\
\hline Calanus spp. CIV & 39 & 56 & 685 & 324 & 638 & 477 & - & - & 266 & 403 & 601 & 640 & 3393 & 10090 & 451 & 323 \\
\hline C. finmarchicus CV & 19 & 10 & 483 & 547 & 133 & 59 & 1 & 2 & 176 & 85 & 238 & 310 & 296 & 8707 & 3 & - \\
\hline C. finmarchicus male & 1 & 1 & 132 & 164 & 11 & - & 1 & 0 & 24 & 17 & 106 & 65 & 4 & 1353 & 38 & 45 \\
\hline C. finmarchicus female & 6 & 5 & 136 & 379 & 71 & - & 50 & 33 & 122 & 43 & 243 & 478 & 97 & 7196 & 14 & 17 \\
\hline C. helgolandicus CV & 2 & 1 & 209 & 350 & 717 & 285 & - & - & 31 & 11 & 74 & 129 & 12 & 466 & 369 & 227 \\
\hline C. helgolandicus male & 0 & - & 60 & 51 & 116 & 91 & - & - & 1 & 5 & 41 & 64 & 1 & 971 & 78 & 48 \\
\hline $\begin{array}{l}\text { C. helgolandicus } \\
\text { female }\end{array}$ & 13 & 1 & 42 & 158 & 205 & 73 & 12 & 23 & 54 & 25 & 58 & 164 & 7 & 1255 & 135 & 190 \\
\hline $\begin{array}{l}\text { Para/Pseudocalanus } \\
\text { spp. I-IV }\end{array}$ & 7557 & 7613 & 2590 & 5055 & 6349 & 29448 & 659 & 809 & 11320 & 17338 & 11941 & 8981 & 100363 & 33547 & 14306 & 11009 \\
\hline Paracalanus parvus V & 7434 & 5347 & 562 & 1687 & 185 & 1534 & 1400 & 301 & 16364 & 10034 & 3830 & 7475 & 3146 & 968 & 41 & 25 \\
\hline P. parvus VI & 3242 & 7100 & 1436 & 2352 & 381 & 1054 & 2635 & 636 & 16264 & 12976 & 11888 & 28986 & 10061 & 17284 & 43 & 101 \\
\hline $\begin{array}{l}\text { Pseudocalanus } \\
\text { elongatus } \mathrm{V}\end{array}$ & 978 & 949 & 2212 & 1604 & 5064 & 12292 & 44 & 125 & 2068 & 3405 & 1254 & 2595 & 17185 & 12489 & 2008 & 1305 \\
\hline P. elongatus VI & 708 & 807 & 4643 & 2999 & 11520 & 13048 & 528 & 686 & 4547 & 3609 & 4776 & 4115 & 16003 & 33290 & 4285 & 3768 \\
\hline $\begin{array}{l}\text { Temora longicornis } \\
\text { CI-CIV }\end{array}$ & 1704 & 1078 & 2060 & 2945 & 7866 & 21595 & - & - & 1480 & 2447 & 5687 & 3713 & 8090 & 9004 & 54 & 50 \\
\hline T. longicornis CV & 431 & 146 & 1382 & 1791 & 4942 & 8489 & - & - & 161 & 344 & 578 & 522 & 4519 & 3235 & - & - \\
\hline T. longicornis CVI & 329 & 174 & 3088 & 1147 & 16306 & 13668 & - & - & 390 & 223 & 235 & 996 & 1779 & 5839 & 72 & 240 \\
\hline Centropages spp. & - & 63 & 1201 & 445 & 1454 & 1938 & 67 & 23 & 297 & 98 & 551 & 1074 & 4179 & 521 & 12 & 26 \\
\hline Metridia lucens & 50 & 1 & 134 & 176 & 87 & - & 1 & 1 & 313 & 122 & 169 & 162 & 821 & 459 & 3 & - \\
\hline Oithona spp. & 7144 & 5631 & 978 & 1589 & 1650 & 8215 & 4023 & 4002 & 24615 & 18205 & 12225 & 8496 & 63606 & 41299 & 7050 & 3906 \\
\hline Harpacticoid copepods & ls 43 & 54 & 9 & 92 & 316 & 4583 & 27 & 143 & 25 & 30 & 80 & 254 & & 143 & 277 & 406 \\
\hline Copepod nauplii & 1107 & 1341 & 393 & 368 & 1260 & 7569 & 123 & 129 & 1050 & 1866 & 5749 & 2185 & 11294 & 3649 & 1328 & 687 \\
\hline Euphausiid calyptopis & 21 & 75 & 485 & 247 & - & 1 & - & - & 27 & 83 & 807 & 341 & 132 & 197 & 101 & 9 \\
\hline Evadne spp. & - & 29 & 398 & 1115 & 5888 & 1440 & 4 & - & 95 & & 968 & 2346 & 22605 & 3526 & - & 47 \\
\hline Podon spp. & - & 15 & - & 44 & 218 & 107 & - & 13 & 12 & 47 & 54 & 95 & 285 & - & - & - \\
\hline Appendicularia & 1942 & 3224 & 1088 & 1876 & 915 & 836 & 1273 & 1299 & 4024 & 6617 & 2254 & 1432 & 1684 & 276 & 2777 & 1202 \\
\hline Cirripede nauplii & 8241 & 2983 & 3249 & 3765 & 579 & 2232 & 19 & 15 & 1116 & 4184 & 3893 & 11202 & - & - & 4659 & 7045 \\
\hline Cirripede cypris & - & 8 & 93 & 32 & 510 & 2342 & - & - & 68 & 44 & 118 & 71 & 681 & 61 & 1452 & 1731 \\
\hline Echinoderm larvae & 3378 & 9992 & 192 & 336 & 46 & - & 9653 & 7650 & 29291 & 35933 & 6640 & 6461 & 493 & - & 3455 & 2359 \\
\hline Polychaete larvae & 75 & 154 & 189 & 270 & 112 & 523 & 1108 & 587 & 741 & 804 & 280 & 685 & 99 & - & 820 & 408 \\
\hline Gastropod larvae & 150 & 27 & - & 14 & 66 & - & 24 & 4 & 95 & - & 12 & - & - & - & 145 & 136 \\
\hline Lamellibranch larvae & 127 & 160 & 33 & 24 & - & 48 & 115 & 169 & 210 & 275 & 138 & 176 & 796 & 424 & 467 & 148 \\
\hline Decapod larvae & 21 & 30 & 157 & 119 & 510 & 429 & 10 & 3 & 49 & 57 & 443 & 139 & 22 & 27 & 262 & 349 \\
\hline Cyphonautes larvae & 436 & 813 & 114 & 240 & 211 & 333 & 1272 & 1086 & 18502 & 23287 & 1713 & 3419 & 1322 & 2261 & 5369 & 2800 \\
\hline Limacina spp. & 14 & 197 & 151 & 168 & - & - & 61 & - & 92 & 134 & 150 & 192 & & & & \\
\hline Tomopteris spp. & 2 & 0 & 1 & 4 & - & - & 8 & 31 & 15 & 42 & 23 & 28 & 0 & 53 & 10 & 10 \\
\hline $\begin{array}{l}\text { Chaetognaths } \\
\text { (Sagitta spp.) }\end{array}$ & 22 & 31 & 131 & 61 & 14 & 16 & 204 & 114 & 160 & 52 & 109 & 75 & 342 & 528 & 43 & 32 \\
\hline Coelenterata & 2 & 6 & 1 & 1 & 46 & 37 & 12 & 29 & 13 & 8 & 4 & 10 & 20 & 52 & 78 & 28 \\
\hline Fish larvae & 11 & 6 & 24 & 69 & 44 & 107 & 1 & 1 & 10 & 15 & 31 & 18 & 2 & 3 & 5 & 8 \\
\hline Others & 248 & 289 & 386 & 359 & 337 & 158 & 18 & 140 & 1074 & 1228 & 5585 & 4571 & 2507 & 3751 & 720 & 1186 \\
\hline Total & 466325 & 50979 & 32668 & 35714 & 70704 & 136445 & 23385 & 18152 & 137476 & 147886 & 92836 & 113539 & 386892 & 378065 & 51672 & 40684 \\
\hline
\end{tabular}

zons) between day and night, and they were therefore considered to perform little or no DVM (Fig. 4). Only on 2 occasions did a significant proportion of the population perform DVM: on 25 May 1988 in the central Irish Sea, when the thermocline was well established, and on 22 April 1989, when the population was dominated by Pseudocalanus elongatus (Fig. 4, Table 2). 
None of the most abundant meroplanktonic larvae (cirripede nauplii and echinoderm larvae) showed marked DVM, whereas holoplanktonic organisms displayed different patterns. Copepods exhibited a range of DVM behaviour: some, such as Acartia spp., Temora longicornis, Oithona spp. and copepod nauplii showed little or no DVM; however, as mentioned earlier, our methodology would underestimate migration amplitude if midnight sinking (Ashjian et al. 1998) or twilight migration (e.g. as sometimes reported for Temora sp.: Pearre 2000) occurs. Pseudocalanus elongatus and Calanus spp. showed a range of DVM amplitudes, including very clear reverse migration (moving deeper during the night) for Calanus spp. in the coastal area.

Appendicularians did not usually undergo DVM, except in the central Irish Sea during May 1988. At this time the thermocline was well established and a strong reverse appendicularian DVM was recorded. Other organisms that moved deeper during the night at the stratified station were the copepods Paracalanus parvus, Metridia lucens and Oithona spp., and cypho- nautes larvae (Table 3). However, an abundant holoplanktonic non-copepod genus in the May 1988 samples, the cladocerans Evadne spp., showed no DVM behaviour, nor did the most abundant organisms at that time, copepods of the genus Acartia.

Among the predators, there was a clear-cut difference between chaetognaths (Sagitta spp.), which performed extensive DVM, and fish larvae that did not seem to migrate vertically (Fig. 3 ).

\section{Calanus spp.}

We paid particular attention to Calanus spp. for 4 reasons: (1) in the initial analysis Calanus spp. shows a very high variability in DVM, including a clear reverse $\mathrm{DVM}_{i}(2)$ the large size of this copepod allows easy identification of the stages and therefore an ontogenetic analysis of its DVM behaviour; (3) the simultaneous presence of $C$. finmarchicus and $C$. helgolandicus allowed detailed comparison between closely

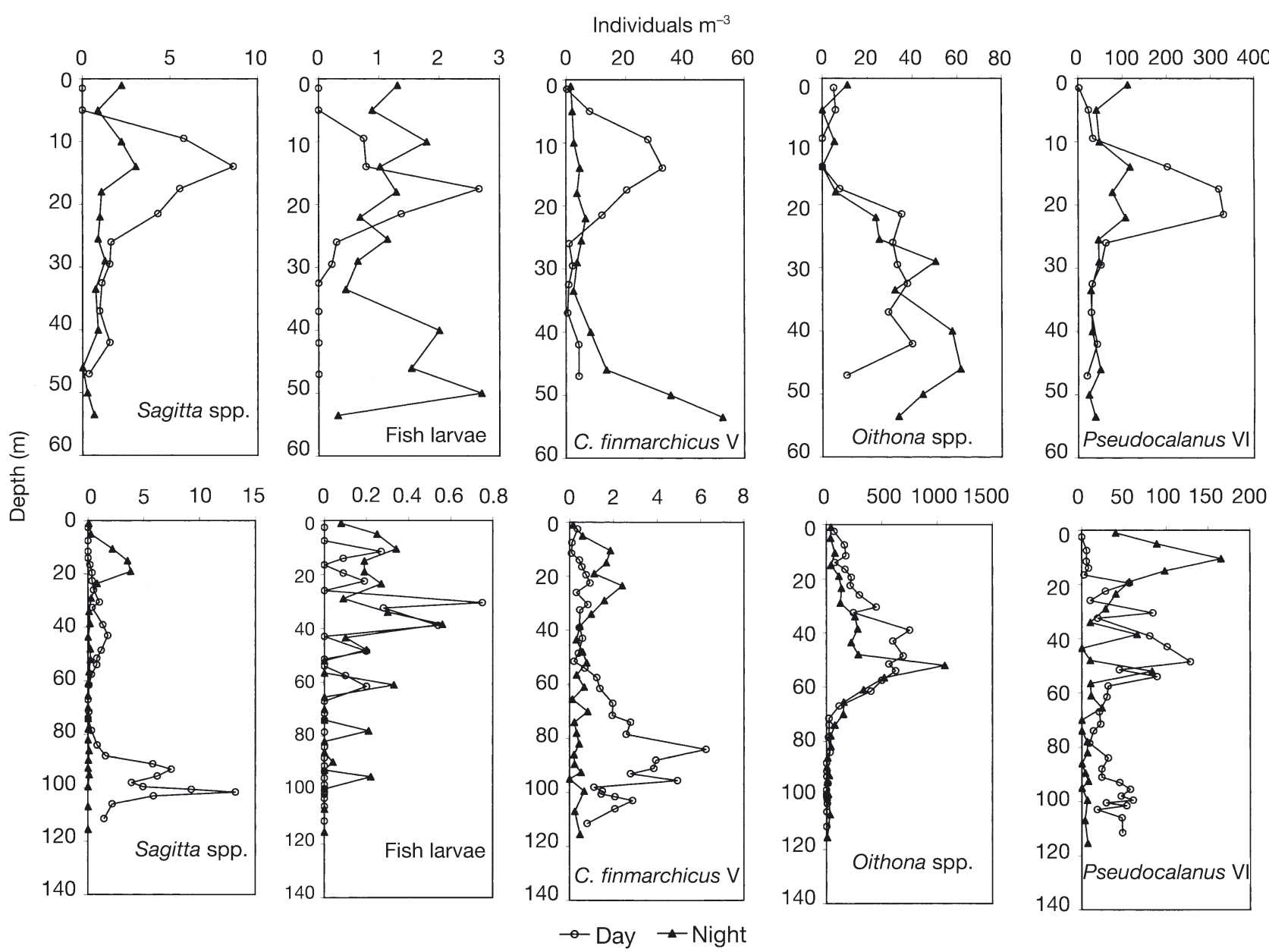

Fig. 2. Examples of vertical distribution of some of main zooplankton groups. Upper graphs = coastal (11 April 1988) and lower graphs $=$ central Irish Sea (11 April 1987). C. = Calanus; Pseudocalanus $=$ P. elongatus 


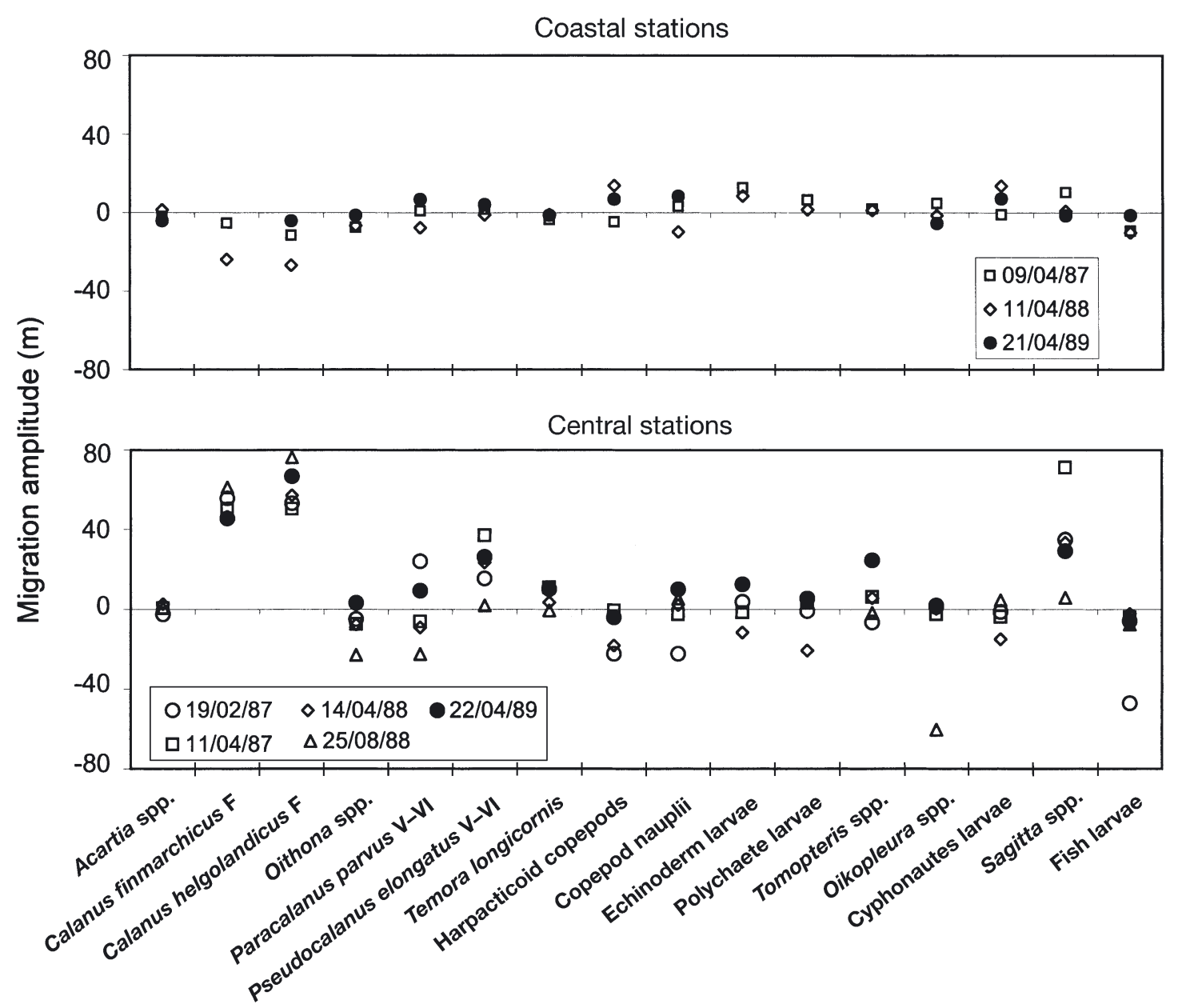

Fig. 3. Examples of amplitude of migration of main components of zooplankton at coastal and central Irish Sea stations. Negative values indicate reverse migration. F: female; V-VI: Copepodite stages V-VI

related species; (4) previous investigations on the vertical distribution of $C$. finmarchicus and C. helgolandicus had been carried out in the adjoining Celtic Sea (Williams 1985).

The DVM behaviour of Calanus spp. differed markedly between the coastal and central areas of the Irish Sea. In the central region, $C$. finmarchicus and $C$. helgolandicus performed normal DVM (migrating to the surface at night), whereas at the coastal station both species performed reverse vertical migration (moving deeper at night) (Fig. 5). Both normal and reverse DVM displayed a clear ontogenetic trend, with the amplitude of DVM increasing with the increasing copepodite stage until Stage V (Fig. 5). There were no significant differences in DVM amplitude (Fig. 5) or vertical position (WMD) between C. finmarchicus and C. helgolandicus, even during the period of stratification (see example for Stage V in Fig. 6). This contrasts with the behaviour recorded by Williams (1985) in the Celtic Sea.

\section{Relations between prey WMD and DVM and predator abundance and behaviour}

Correlation coefficients between the WMD of different organisms were used to analyse possible predator-prey relationships (Table 4). Cells of the large diatom Coscinodiscus spp., also collected by the net, were included in the analysis as a passive tracer. Although Coscinodiscus spp. have been observed to undergo reverse migrations (Hardy \& Gunther 1935), the amplitude of such migrations is smaller than that of the zooplankton. The highest correlation for chaetognath WMD was with the WMD of Pseudocalanus elongatus $(\mathrm{r}=0.79)$, followed by various stages of Calanus spp. ( $\mathrm{r}=0.51$ to 0.78 ) (Fig. 7). For fish larvae, the highest correlation was with Oithona spp. $(\mathrm{r}=0.80)$ and copepod nauplii ( $\mathrm{r}=0.76)$ (Fig. 7).

The amplitude of the DVM of 3 of the most actively migrating copepods (Calanus finmarchicus, C. hel- 
golandicus and Pseudocalanus elongatus) was compared with the abundance and position in the water column of the chaetognaths. For all 3 copepods the only significant relationship was with the WMD of chaetognaths during the day (Fig. 8). The extreme chaetognath WMD of $90 \mathrm{~m}$ influenced the significance of the obtained relationships; if this is considered an outlier, then the relationship between $P$. elongatus DVM and Sagitta spp. WMD becomes non-significant, whereas the relation between $C$. helgolandicus V DVM and Sagitta spp. WMD becomes significant $\left(\mathrm{r}^{2}=\right.$ $0.59, \mathrm{p}<0.05)$.

Table 3. Daytime weighted mean depth (WMD, m) and amplitude of diel vertical migration (DVM, m) of main zooplankton groups at coastal and central Irish Sea stations. Negative DVM values indicate reverse migration (deeper at nighttime than during the day)

\begin{tabular}{|c|c|c|c|c|c|c|c|c|c|c|c|c|c|c|c|c|}
\hline \multirow[b]{2}{*}{ Acartia spp. I-IV } & \multicolumn{2}{|c|}{$\begin{array}{c}\text { 09/04/87 } \\
\text { WMD DVM }\end{array}$} & \multicolumn{2}{|c|}{$\begin{array}{c}\text { Coastal } \\
\text { 11/04/88 } \\
\text { WMD DVM }\end{array}$} & \multicolumn{2}{|c|}{$\begin{array}{c}21 / 04 / 89 \\
\text { WMD DVM }\end{array}$} & \multicolumn{2}{|c|}{$\begin{array}{c}12 / 02 / 87 \\
\text { WMD DVM }\end{array}$} & \multicolumn{2}{|c|}{$\begin{array}{c}11 / 04 / 87 \\
\text { WMD DVM }\end{array}$} & \multicolumn{2}{|c|}{ 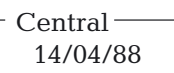 } & \multicolumn{2}{|c|}{$\begin{array}{c}\text { 25/05/88 } \\
\text { WMD DVM }\end{array}$} & \multicolumn{2}{|c|}{$\begin{array}{c}22 / 04 / 89 \\
\text { WMD DVM }\end{array}$} \\
\hline & 7.7 & -2.9 & 10.7 & 6.2 & 4.2 & -11.3 & $12.5-$ & -24.9 & 19.3 & 1.1 & 15.2 & 2.5 & 10.3 & -1.5 & 11.0 & -7.4 \\
\hline Acartia spp. V & 5.2 & -5.6 & 7.6 & -0.7 & 12.1 & 9.0 & - & - & 16.8 & -0.9 & 13.9 & 2.3 & 12.1 & 0.8 & & \\
\hline Acartia spp. VI & 12.6 & 3.0 & 8.7 & -0.5 & 12.3 & -4.1 & 45.4 & 14.4 & 17.7 & 0.8 & 12.5 & 2.3 & 21.0 & 8.1 & 44.2 & 21.2 \\
\hline Calanus spp. CI & 13 & 2.2 & 11.9 & -4.6 & 21.5 & 15.7 & - & - & 27.4 & -0.4 & 21.1 & 4.8 & 19.6 & 11.1 & 20.8 & 11.6 \\
\hline Calanus spp. CII & 13.8 & 0.3 & 11.7 & -4.9 & 12.1 & -2.1 & - & - & 29.3 & 1.3 & 24.8 & 8.9 & 32.3 & 20.9 & 21.0 & 11.5 \\
\hline Calanus spp. CIII & 11.2 & 0.2 & 11.4 & -14.5 & 8.8 & -1.3 & 94.2 & - & 31.4 & -2.4 & 44.1 & 16.4 & 42.2 & 31.0 & 31.5 & 18.9 \\
\hline Calanus spp. CIV & 9.8 & -7.9 & 14.4 & -10.2 & 8.3 & -3.4 & - & - & 63.0 & 31.3 & 63.5 & 24.9 & 55.2 & 40.3 & 65.4 & 54.8 \\
\hline C. finmarchicus CV & 13.5 & -6.8 & 16.6 & -26.8 & 8.8 & -8.1 & 51.7 & 16.7 & 80.1 & 38.4 & 81.7 & 39.3 & 65.3 & 43.3 & 49.7 & \\
\hline C. finmarchicus male & 12.3 & -0.9 & 19.9 & -24.1 & 11.0 & - & 77.3 & 63.6 & 85.8 & 5.2 & 79.0 & 18.6 & 75.5 & 7.2 & 75.7 & 72.7 \\
\hline $\begin{array}{l}\text { C. finmarchicus } \\
\text { female }\end{array}$ & 15.3 & -5.4 & 19.8 & -23.9 & 6.5 & - & 75.6 & 55.6 & 83.4 & 50.5 & 79.3 & 46.5 & 82.4 & 61.2 & 59.5 & 45.4 \\
\hline C. helgolandicus CV & 14.1 & 3.3 & 15.1 & -23.3 & 6.2 & -5.3 & - & - & 87.8 & 50.3 & 83.5 & 51.8 & 43.6 & 22.0 & 73.4 & 62.3 \\
\hline C. helgolandicus male & 33.7 & - & 18.1 & -28.8 & 7.2 & -3.7 & - & - & 83.3 & 22.0 & 88.1 & 37.9 & 95.6 & 51.6 & 77.2 & 57.0 \\
\hline $\begin{array}{l}\text { C. helgolandicus } \\
\text { female }\end{array}$ & 6 & -11.7 & 15.2 & -26.8 & 6.8 & -4.2 & 86.7 & 53.1 & 89.2 & 50.4 & 86.0 & 57.1 & 95.0 & 76.4 & 79.1 & 66.8 \\
\hline Paracalanus parvus V & 14.9 & 1.1 & 33.5 & -4.0 & 20.2 & 11.8 & 51.9 & 28.5 & 56.8 & -7.1 & 57.5 & -12.9 & 26.8 & -50.3 & 13.1 & 12.1 \\
\hline P. parvus VI & 17 & 1.5 & 32.1 & -10.1 & 15.2 & 2.3 & 53.6 & 21.9 & 59.6 & -5.1 & 62.7 & -7.8 & 49.6 & -15.7 & 18.0 & 9.9 \\
\hline $\begin{array}{l}\text { Pseudocalanus } \\
\text { elongatus } \mathrm{V}\end{array}$ & 13.9 & 2.3 & 17.1 & -2.1 & 15.8 & 5.3 & 33.8 & 19.2 & 46.1 & 29.5 & 46.6 & 21.4 & 32.5 & 6.9 & 28.8 & 15.3 \\
\hline P. elongatus VI & 15 & 1.1 & 20.8 & -0.7 & 14.9 & 3.2 & 49.4 & 12.4 & 64.6 & 37.5 & 75.1 & 17.5 & 36.1 & 0.8 & 52.7 & 31.6 \\
\hline $\begin{array}{l}\text { Temora longicornis } \\
\text { CI-CIV }\end{array}$ & 14.2 & -4.6 & - & - & 21.0 & 4.4 & - & - & 27.5 & 15.3 & 17.1 & 3.9 & 14.1 & -0.4 & 17.0 & 16.0 \\
\hline T. longicornis CV & 15.9 & 3.6 & 19.2 & -0.6 & 18.9 & -1.2 & - & - & 27.2 & 10.4 & 16.4 & -0.1 & 16.4 & 4.0 & 20.0 & 10.1 \\
\hline T. longicornis CVI & 14.3 & -3.3 & 18.8 & -5.5 & 15.0 & -6.8 & - & - & 22.2 & 10.9 & 17.8 & 3.6 & 26.4 & 1.4 & 23.8 & 11.1 \\
\hline Centropages spp. & - & - & 9.0 & -5.1 & 12.5 & -5.1 & $28.8-$ & -33.3 & 38.0 & -5.0 & 14.6 & 2.7 & 12.4 & -3.8 & 56.5 & - \\
\hline Metridia lucens & 18 & - & 31.4 & -4.8 & 18.4 & - & 85.3 & 41.0 & 93.5 & 67.9 & 83.3 & 31.2 & 40.5 & -34.9 & 36.0 & - \\
\hline Oithona spp. & 11.4 & -7.5 & 30.6 & -6.6 & 12.7 & -1.4 & 33.2 & -4.9 & 41.8 & -7.4 & 27.8 & -7.5 & 17.8 & -22.8 & 32.0 & 3.2 \\
\hline Harpacticoid copepods & 14.5 & -4.8 & 40.6 & 13.9 & 22.4 & 6.8 & $54.4-$ & -22.4 & 94.9 & -0.7 & 51.3 & -18.2 & & 51.1 & -4.1 & \\
\hline Copepod nauplii & 16.7 & 3.3 & 15.3 & -9.8 & 16.2 & 8.4 & 15.7 & -22.4 & 33.0 & -2.6 & 16.2 & 2.2 & 12.0 & 5.4 & 19.8 & 9.9 \\
\hline Euphausiid calyptopis & 12 & -0.5 & 17.1 & -6.6 & - & - & - & - & $39.1-$ & -11.1 & 33.4 & -34.4 & 31.5 & -6.9 & 45.3 & -11.2 \\
\hline Evadne spp. & - & - & 8.6 & -9.3 & 5.6 & -6.5 & 67.5 & 46.5 & 20.9 & - & 10.8 & 1.3 & 9.3 & -1.1 & - & - \\
\hline Podon spp. & - & - & - & - & 14.1 & 1.1 & - & - & 47.1 & 8.6 & 22.4 & 18.4 & - & - & - & - \\
\hline Appendicularia & 14.1 & 4.7 & 18.5 & -1.5 & 6.4 & -5.5 & 42.1 & 1.8 & 37.6 & -2.5 & 14.1 & 0.4 & 16.3 & -60.3 & 17.8 & 1.2 \\
\hline Cirripede nauplii & 12.2 & 0.3 & 9.5 & -1.6 & 23.3 & 15.4 & 44.1 & -9.7 & 35.8 & 6.5 & 23.3 & 4.7 & - & - & 27.2 & 10.0 \\
\hline Cirripede cypris & 24.9 & 12.8 & 23.8 & 8.5 & - & - & 48.8 & 3.6 & 23.9 & -1.5 & 15.5 & -11.7 & 28.9 & 27.4 & 12.5 & \\
\hline Echinoderm larvae & 15.2 & 6.5 & 29.3 & 1.5 & 26.1 & 8.8 & 59.2 & -0.9 & 52.2 & 3.4 & 23.2 & -20.7 & - & - & 39.2 & 5.3 \\
\hline Polychaete larvae & 12.3 & 5.1 & - & - & - & - & $40.9-$ & -32.8 & 47.7 & - & 4.5 & - & - & - & 45.4 & 28.8 \\
\hline Gastropod larvae & 19.5 & 9.5 & 10.8 & -7.3 & - & - & 47.1 & 25.3 & 22.4 & 3.8 & 28.4 & -17.8 & 11.9 & -2.2 & 24.7 & 1.1 \\
\hline Lamellibranch larvae & 7.7 & -12.7 & 14.5 & -11.6 & - & - & 78.3 & 2.7 & 40.0 & -17.1 & 27.1 & -19.1 & 26.2 & -16.5 & 38.0 & -29.8 \\
\hline Decapod larvae & 11.3 & -1.0 & 23.0 & 13.7 & 24.8 & 9.0 & 57.4 & -1.3 & 56.7 & -3.8 & 34.0 & -15.0 & 37.2 & -34.8 & 24.7 & 13.0 \\
\hline Cyphonautes larvae & - & 13.0 & -32.0 & 19.1 & 7.1 & - & - & 75.0 & 14.0 & 44.1 & -43.7 & 26.9 & 4.6 & 50.4 & 12.3 & \\
\hline Limacina spp. & 1 & -19.0 & 12.6 & -1.2 & - & - & 41.3 & - & 69.5 & 30.2 & 43.2 & 20.0 & - & - & - & - \\
\hline Tomopteris spp. & 16.6 & 1.9 & 11.9 & 1.3 & - & - & 40.6 & -6.6 & 39.8 & 6.3 & 44.4 & 5.6 & 36.0 & -1.9 & 51.4 & 24.6 \\
\hline $\begin{array}{l}\text { Chaetognaths } \\
\text { (Sagitta spp.) }\end{array}$ & 21 & 10.3 & 19.3 & 0.8 & 16.0 & -1.6 & 54.5 & 34.9 & 90.9 & 71.3 & 47.8 & 33.4 & 41.7 & 5.8 & 35.7 & 29.2 \\
\hline Coelenterata & 5.8 & -2.7 & 42.0 & 8.5 & 18.8 & 5.9 & 44.6 & -1.9 & 45.8 & -1.9 & 76.3 & 8.8 & 57.9 & -1.9 & 42.0 & 22.3 \\
\hline Fish larvae & 11.1 & -9.3 & 17.8 & -10.2 & 15.4 & -1.5 & $15.0-$ & -47.1 & 33.6 & 4.0 & 18.1 & -2.3 & 11.6 & 7.5 & 22.6 & -5.9 \\
\hline
\end{tabular}



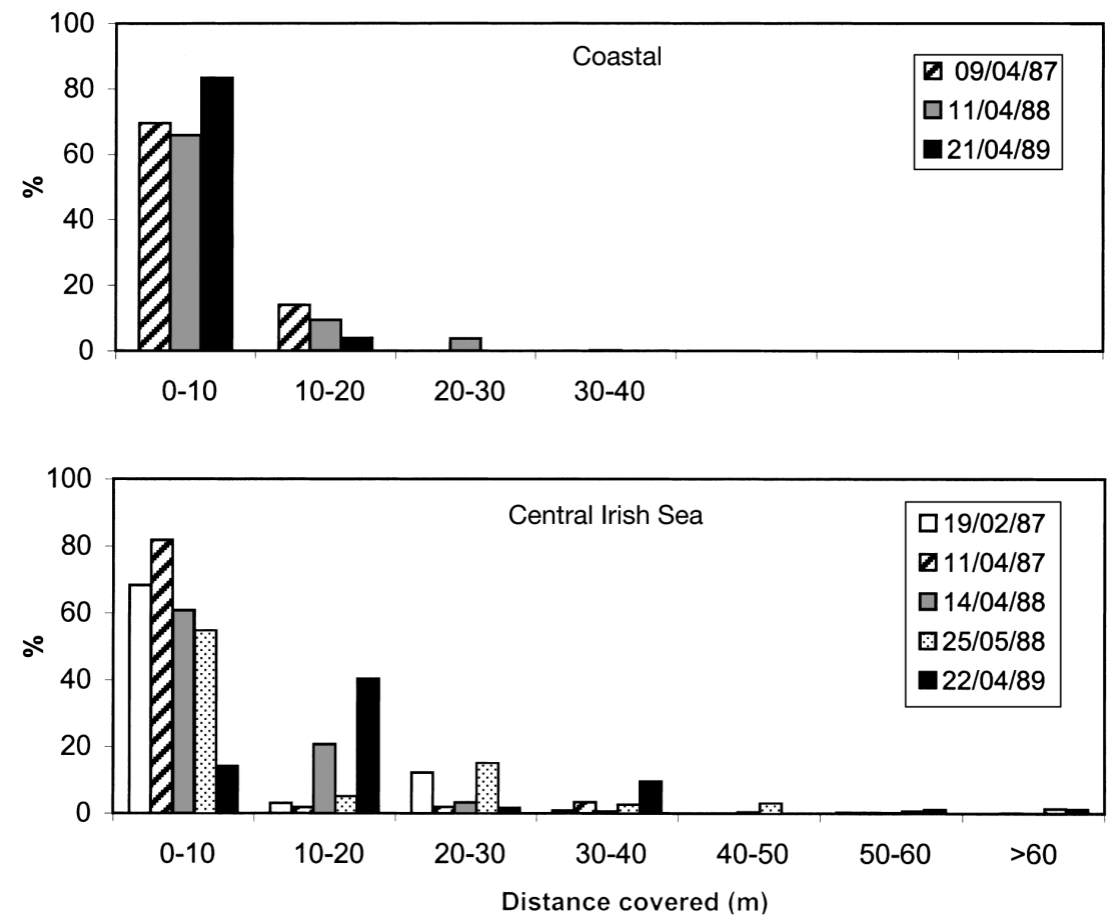

Fig. 4. Distribution (\%) of zooplankton population as a function of distance travelled during diel vertical migration
Also (as stated in 'Materials and methods'), the daytime WMD of Sagitta spp. could have been underestimated due to these being on or near the bottom, below the sampling depth range of our net.

Neither chaetognath abundance nor their distribution at night were related to the amplitude of the migration of Calanus spp. or Pseudocalanus elongatus (Fig. 8). When the chaetognaths displayed reduced or no DVM (Table 3) and remained on the surface during the day (coastal stations), Calanus spp. displayed reverse migration and $P$. elongatus did not migrate or displayed limited reverse DVM. When chaetognaths displayed normal DVM (central Irish Sea), both Calanus spp. and $P$. elongatus resumed normal DVM (Fig. 8).

The DVM of the chaetognaths seems to be related to bottom depth (Fig. 9). At the coastal stations, chaetognaths remained 15 to $20 \mathrm{~m}$ above the bottom, with only small differences in their vertical distribution between day and night. In the central Irish Sea, chaetognaths tended to stay deeper during the day and move towards the surface at night.

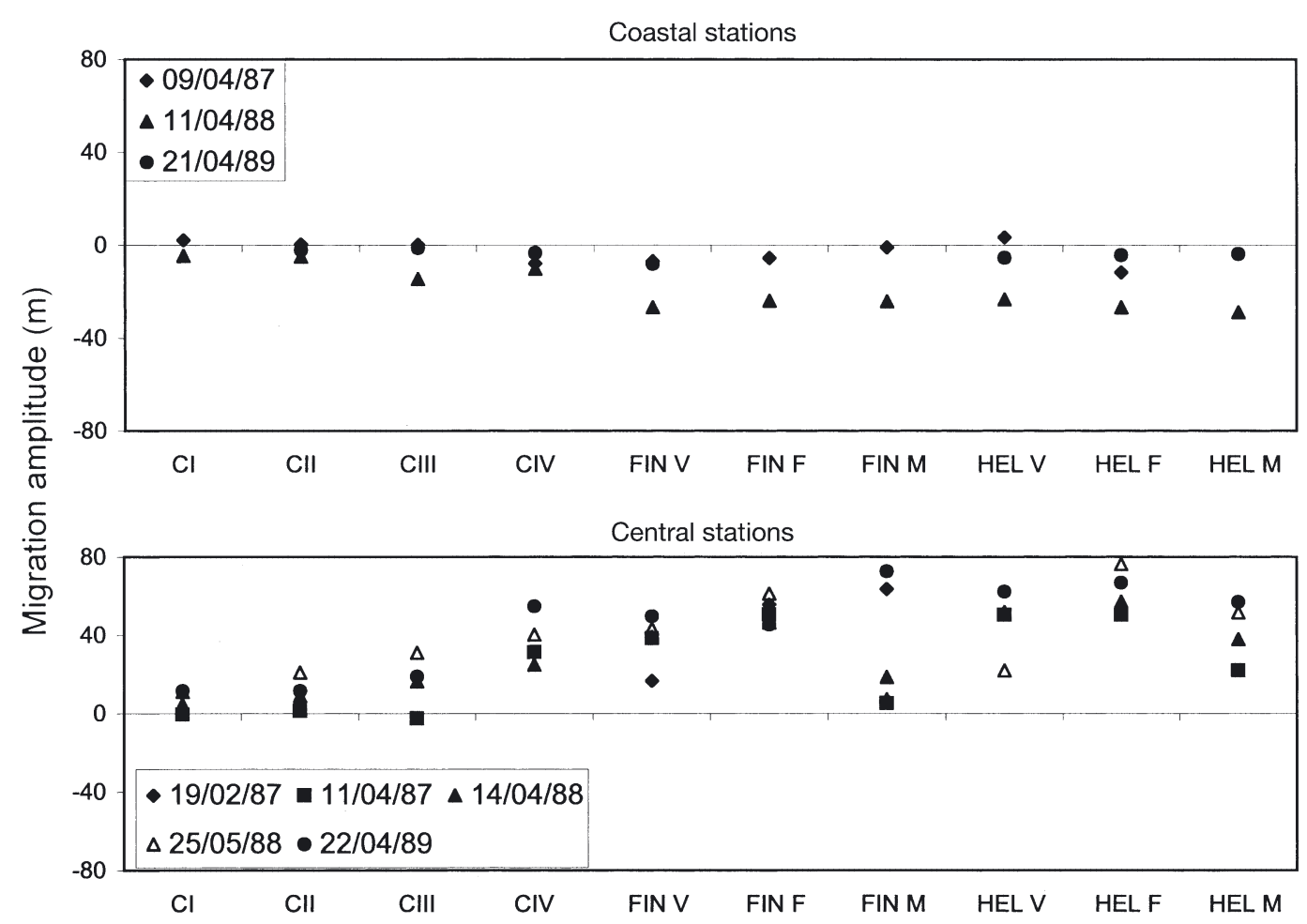

Fig. 5. Calanus spp. Amplitude of diel vertical migration for different copepodite developmental stages (I, II, III, IV, V) at coastal and central Irish Sea stations. F: females; M: males; FIN: C. finmarchicus; HEL: C. helgolandicus 

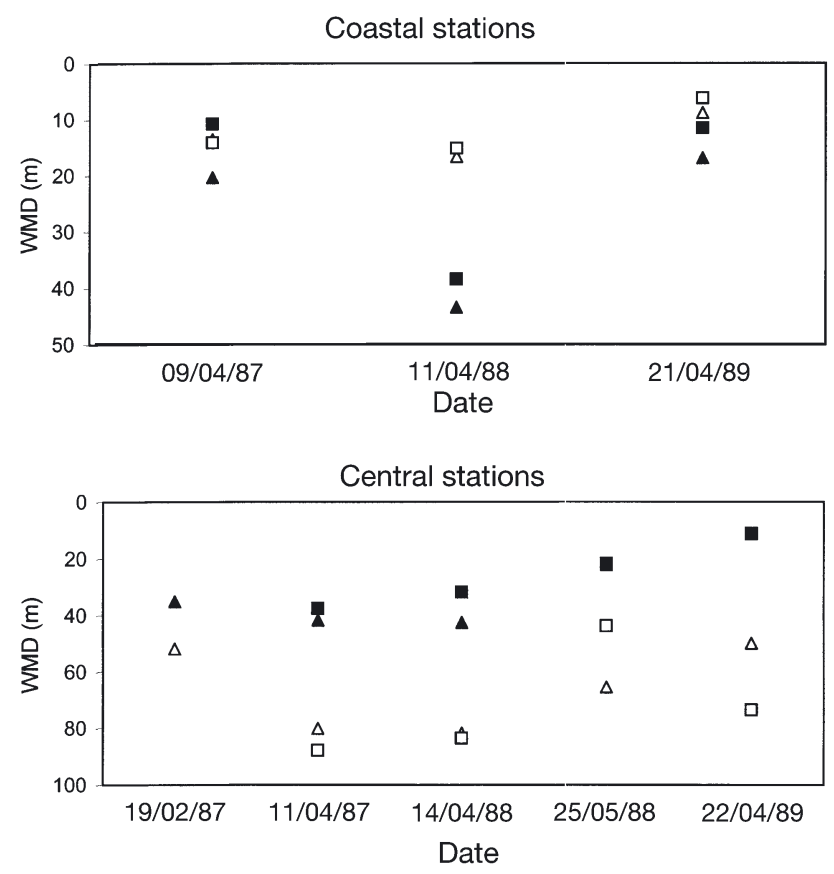

Fig. 6. Calanus spp. Weighted mean depth (WMD) of Copepodite Stage V of C. helgolandicus ( $\mathbf{m}$ : night-time; 口: daytime) and $C$. finmarchicus ( $\mathbf{\Delta}$ : night-time; $\Delta$ : daytime) at coastal and central Irish Sea stations
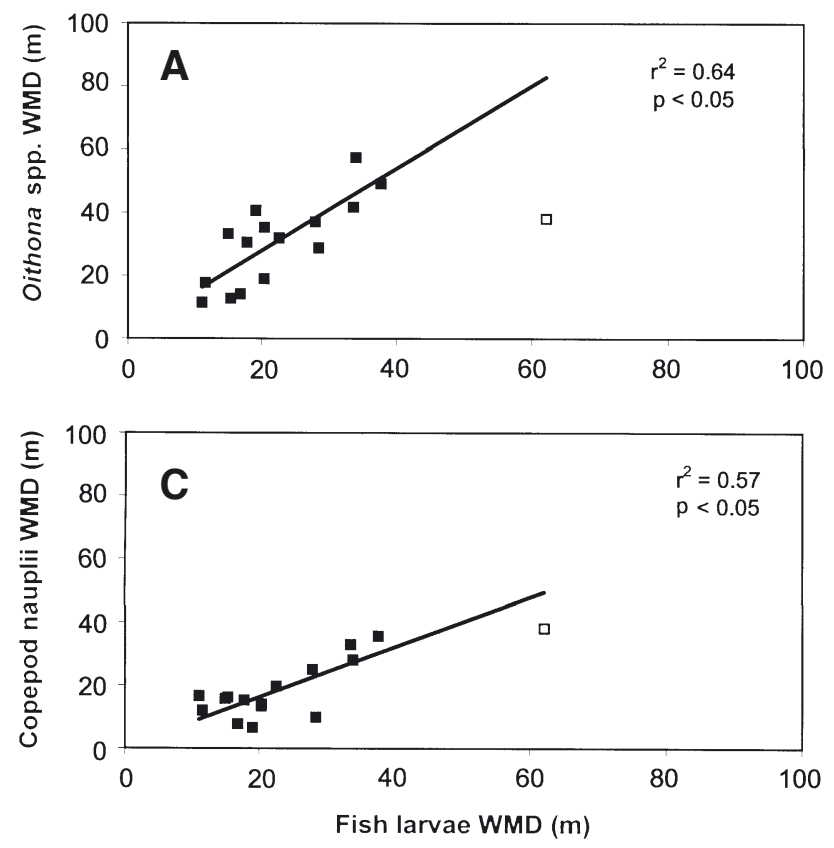

Table 4. Correlation coefficients between weighted mean depth of the main zooplankton groups and 2 potential predators, chaetognaths (Sagitta spp.) and fish larvae. Significant correlation coefficients indicated in bold

\begin{tabular}{|lcc|}
\hline & Sagitta spp. & Fish larvae \\
\hline Acartia spp. I-IV & 0.32 & $\mathbf{0 . 5 8}$ \\
Acartia spp. VI & 0.53 & 0.02 \\
Calanus spp. I & 0.53 & 0.52 \\
Calanus spp. II & $\mathbf{0 . 6 5}$ & 0.38 \\
Calanus spp. III & $\mathbf{0 . 5 1}$ & 0.06 \\
Calanus spp. IV & $\mathbf{0 . 6 7}$ & 0.40 \\
C. finmarchicus V & $\mathbf{0 . 6 9}$ & $\mathbf{0 . 5 4}$ \\
C. finmarchicus female & $\mathbf{0 . 7 6}$ & 0.22 \\
C. finmarchicus male & $\mathbf{0 . 6 8}$ & 0.35 \\
C. helgolandicus V & $\mathbf{0 . 7 8}$ & 0.39 \\
C. helgolandicus female & $\mathbf{0 . 7 1}$ & 0.37 \\
C. helgolandicus male & $\mathbf{0 . 6 4}$ & 0.37 \\
Oithona spp. & 0.32 & $\mathbf{0 . 8 0}$ \\
Paracalanus parvus V-VI & 0.44 & 0.32 \\
Pseudocalanus elongatus V-VI & $\mathbf{0 . 7 9}$ & 0.14 \\
Temora longicornis I-IV & 0.65 & -0.02 \\
T. longicornis V-VI & 0.38 & -0.26 \\
Harpacticoid copepods & 0.45 & $\mathbf{0 . 6 0}$ \\
Copepod nauplii & 0.40 & $\mathbf{0 . 7 6}$ \\
Cirripede nauplii & $\mathbf{0 . 6 6}$ & 0.41 \\
Echinoderm larvae & 0.48 & -0.30 \\
Polychaeta larvae & $\mathbf{0 . 6 3}$ & 0.35 \\
Tomopteris spp. & 0.48 & -0.04 \\
Appendicularia & 0.46 & 0.34 \\
Cyphonautes larvae & 0.52 & 0.28 \\
Coscinodiscus spp. & 0.14 & 0.22 \\
\hline
\end{tabular}
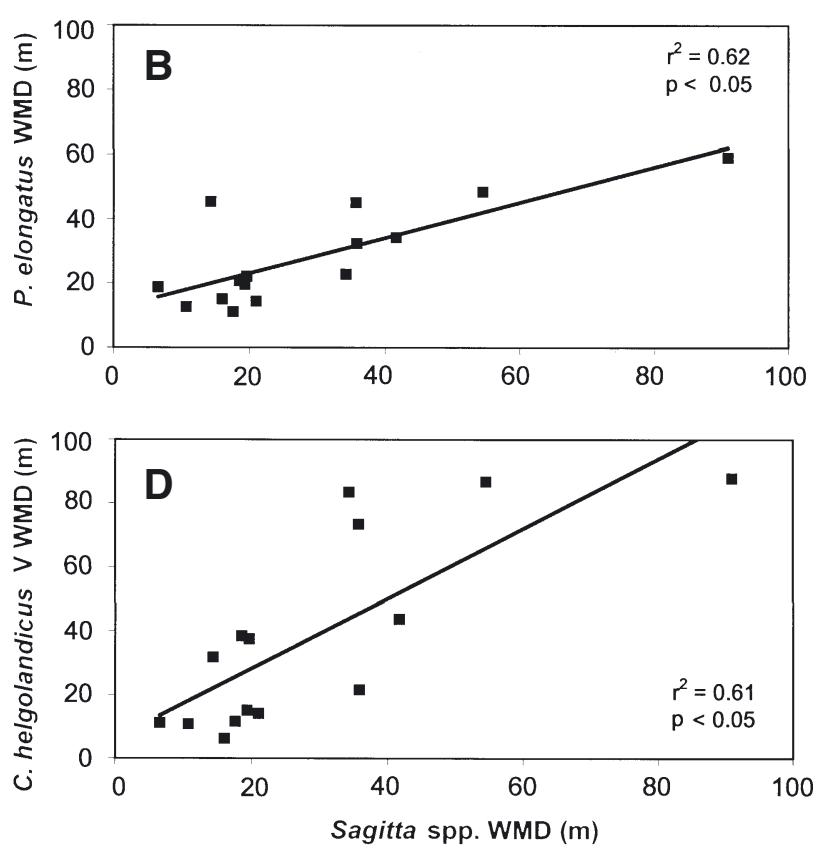

Fig. 7. Examples of significant correlations between prey and their potential predators (see Table 4). (A) Oithona spp. vs fish larvae, (B) Pseudocalanus elongatus vs Sagitta spp., (C) copepod nauplii vs fish larvae, and (D) Calanus helgolandicus Copepodite V vs Sagitta spp.. WMD: Weighted mean depth. Open data points indicate data excluded from correlation analysis 

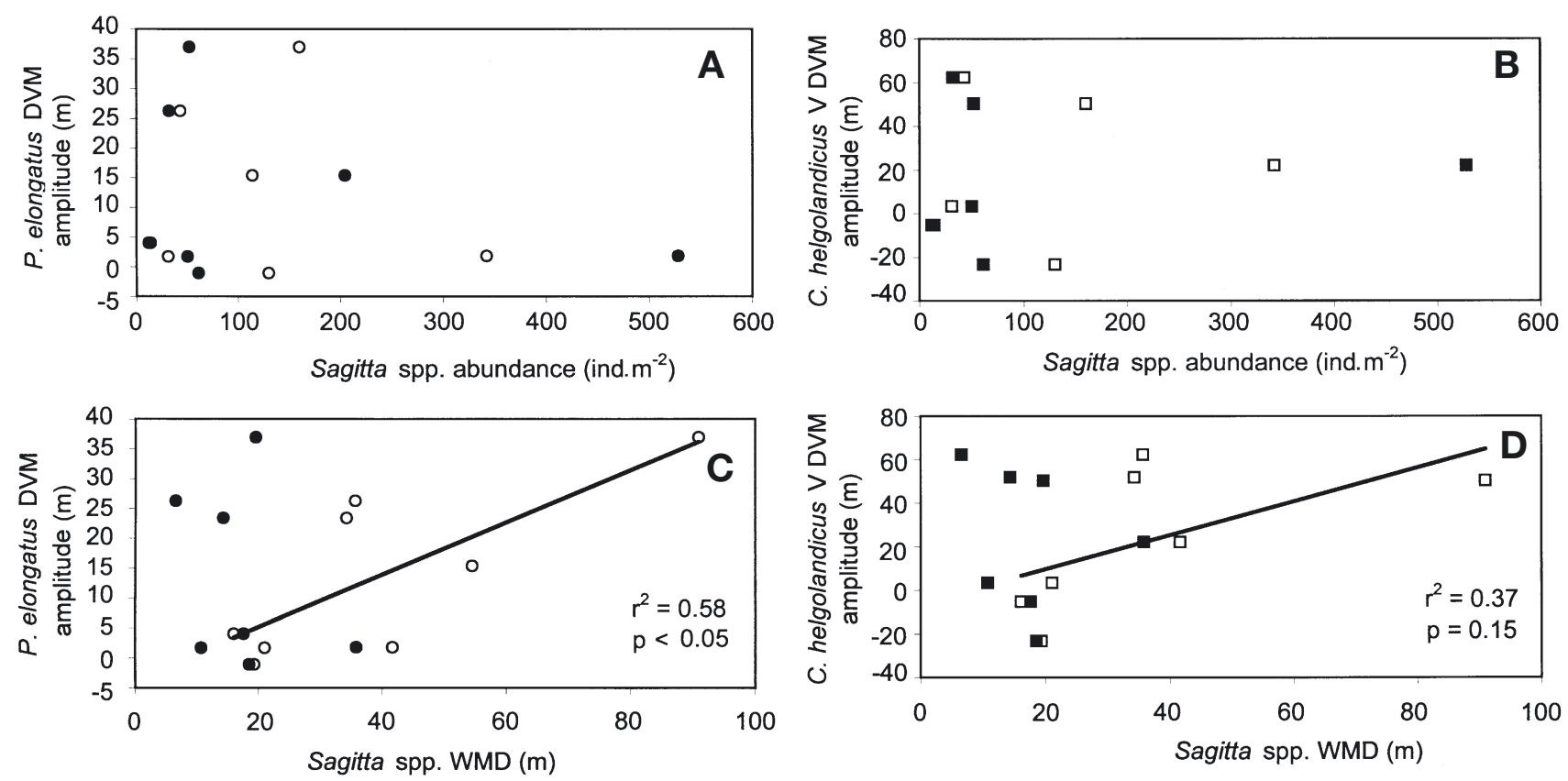

Fig. 8. Relationship between diel vertical migration (DVM) of prey and abundance and weighted mean depth (WMD) of potential predators. Filled symbols indicate night-time and open symbols daytime data for (A) Pseudocalanus elongatus DVM vs Sagitta spp. abundance, (B) Calanus helgolandicus Copepodite V DVM vs Sagitta spp. abundance, (C) P. elongatus DVM vs Sagitta spp. WMD, and (D) C. helgolandicus Copepodite V DVM vs Sagitta spp. WMD

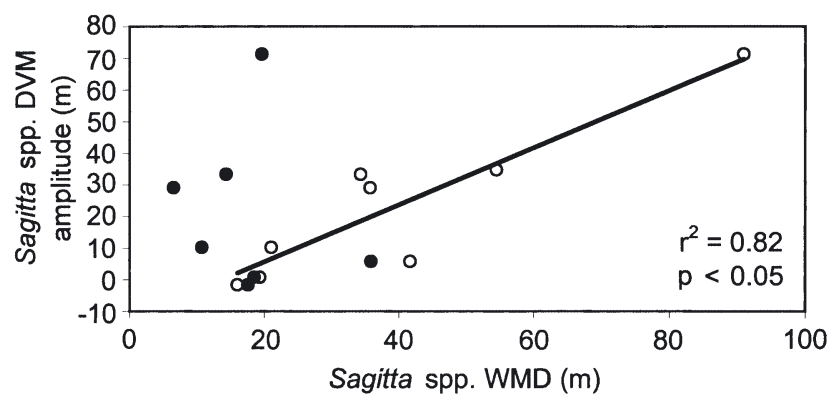

Fig. 9. Relationship between Sagitta spp. diel vertical migration (DVM) and weighted mean depth (WMD). (•) Nighttime data; ( $($ ) daytime data

\section{DISCUSSION}

The most striking result of this study is the high variability in DVM behaviour within the same planktonic community. If we assume that DVM is the result of a compromise between reducing mortality and energy expenditure, and taking advantage of feeding opportunities (Iwasa 1982, Ohman 1990), this variability would seem to have 2 main, partially linked, reasons: (1) the same behaviour does not produce the same optimum compromise between reduced mortality and energetic cost for different groups, species and stages; (2) the mortality risks are not the same for all groups, species or stages, because of the selectivity of predators.
For meroplanktonic larvae, for example, the best way to reduce mortality would probably be to increase growth and reduce time spent in the plankton by increasing their feeding and profiting from the higher temperatures in the upper water layer (Neverman \& Wurtsbaugh 1994), rather than engaging in DVM at the risk of prolonging the planktonic phase.

A similar reason could be responsible for the ontogenic differences in the DVM behaviour of Calanus spp. Ontogenic differences in DVM have been previously reported for C. pacificus in mesocosm experiments (Huntley \& Brooks 1982) and in the field (Osgood \& Frost 1994), C. finmarchicus in Scottish lochs and the Norwegian Sea (Nicholls 1933, Marshall \& Orr 1955, Dale \& Kaartvedt 2000), Metridia lucens in different areas (Osgood \& Frost 1994, Hays et al. 1995, Falkenhaugh et al. 1997, Timonen 1997), and for Neocalanus gracilis, Pleuromamma abdominalis and Euchaeta acuta in the Mediterranean (Andersen et al. 2001).

Ontogenic and size-dependent variations in DVM have generally been attributed to large individuals being more conspicuous and therefore more susceptible to visual predators (Hays et al. 1994, De Robertis et al. 2000) and to size-selectivity (Neill 1992). However, nauplii and early copepodite stages are usually the main food source of fish larvae (e.g. Conway 1980, Conway et al. 1998); they also suffer from invertebrate predation, and they often have higher mortality rates than adults (Ohman et al. 2002). Therefore, one would 
expect them to enhance the behaviours aimed at reducing mortality. Furthermore, Huntley \& Brooks (1992) observed ontogenic differences in the DVM of Calanus pacificus in the absence of predators and found that migration decreased at low food concentrations. It has also been observed that early nauplii of $C$. pacificus do not have the ability to survive long starvation events (Lopez 1996). Therefore, it is possible that for a nauplius or an early copepodite stage, a rapid increase in size will result in a less mortality (Fiksen \& Giske 1995). This would be a better strategy than engaging in DVM. In contrast, for late copepodite stages or adults, in which increased feeding will not result in a significant increase in size, DVM would probably be a better option.

The minimum distance to be covered in order to reduce the risk of predation depends on irradiance, attenuation coefficient, visibility of prey and the visual system of the predator (Aksnes \& Utne 1997). Therefore, the mortality risk/energy gain ratio would be highly dependent on environmental conditions (De Robertis 2002). This mortality risk/energy gain ratio could be particularly important in trying to understand the behaviour of small copepods such as Oithona spp., which do not usually show DVM behaviour. It is possible that for such small copepods the energetic cost of swimming outweighs the reduction in mortality achieved by thus avoiding visual predators, and that other strategies such as reduction of their mechanical signals (Paffenhöfer \& Mazzocchi 2002) are a better option in the balance between reducing mortality and energetic cost. Although it is possible that copepods use an elastin-like protein that would decrease the energetic requirement of vertical displacement (Alcaraz \& Strickler 1988), the energetic side of the equation could be more important than previously thought. Larger copepods such as Metridia pacifica cease migration when their lipid storage is low (Hays et al. 2001), and even for organisms as large as northern krill Meganyctiphanes norvegica, a period in deep waters can result in the breakdown of haemocyanin for use as an energy source in order to maintain basal metabolism (Spicer \& Stromberg 2002).

Whereas Pseudocalanus elongatus performed significant DVM in the central Irish Sea, adults of similarly sized or slightly larger copepods such as Acartia, Temora and Centropages spp. showed much reduced or no apparent DVM. Nevertheless, it is possible that due to the different reproductive strategy of these species (free-egg spawning as opposed to egg-sac spawning in $P$. elongatus), the balance between mortality risk and feeding shifts towards feeding. When food concentrations are high, egg production in free-spawners is proportional to ingestion, and fluctuations in the food supply significantly reduce daily egg production
(Calbet \& Alcaraz 1996). Because in free-spawners the death of the female does not imply the death of the eggs, as is the case with sac-spawners such as $P$. elongatus, it is possible that free-spawners risk higher mortality in order to assure maximum egg production by continuous feeding.

Another possible explanation could be that Acartia spp., Centropages spp. and Temora longicornis are not the main targets of predators in the area. The selective behaviour of predators introduces a further variable into the interpretation of the DVM variability. Analyses of gut contents are needed to confirm whether the relationship we found between copepod species and potential predators is due to preference for those preys (chaetognath WMD-Pseudocalanus elongatus WMD, chaetognath WMD-Calanus spp. WMD, fish larvae WMD-copepod nauplii WMD, fish larvae WMDOithona spp. WMD). However, in the literature, copepod nauplii and Oithona spp. are often reported to be the main prey of fish larvae (Conway 1980, Conway et al. 1998) and copepods the main prey of chaetognaths (Duro \& Saiz 2000), even when large numbers of alternative prey are abundant in the plankton (Saito \& Kiørboe 2001). Chaetognaths are likely to be mainly sizeselective predators (e.g. Pearre 1980), but they also show selection between different copepod species, as well as a flexibility in their selectivity (RakusaSuszczeweski 1969). As an example, in the North Sea, Sagitta elegans showed high feeding rates on male Paracalanus spp., followed by species of Calanus, Metridia, Centropages and Pseudocalanus, whereas the feeding rates on Oithona spp. and on Paracalanus spp. Stages III and IV were much lower (Saito \& Kiørboe 2001).

While, in order to reduce mortality by fish larvae predation, copepod nauplii and Oithona spp. seem to have adopted strategies other than DVM (i.e. rapid growth or 'invisibility'), both Calanus spp. and Pseudocalanus spp. show clear evidence of active avoidance of chaetognaths by adapting their DVM inversely to that of the chaetognaths. However, contrary to the situation found by Ohman (1990), in our study it was apparently not the abundance of chaetognaths that triggered DVM (normal or reverse), but the position of the chaetognaths in the water column during daytime. Calanus spp. and $P$. elongatus appear to sense the position of chaetognaths in the water column during the period of lower feeding activity, possibly using chemical cues (see references in Lampert 1993), and to adapt the amplitude and direction of their DVM accordingly. In the coastal area, because chaetognaths do not migrate as close to the bottom during the day, this results in reverse DVM for Calanus spp. and slightly reversed or no migration for $P$. elongatus. Again, even if the answer is similar, the difference in 
the migration amplitude of Calanus spp. and P. elongatus could be due to differences in the energetic cost of swimming for large and small copepod species.

Many uncertainties and questions remain. For example, why do Paracalanus parvus apparently not use DVM to avoid chaetognaths? Would chaetognaths have a greater preference for Pseudocalanus spp. rather than Paracalanus spp. (these 2 genera are very similar in the early stages)? Chaetognaths do show preferences, not only as a function of prey size, but also as a function of the hydrodynamic signal produced (Saito \& Kiørboe 2001). Data from Dabob Bay and the North Sea (Ohman 1986, Saito \& Kiørboe 2001) seem to indicate a preference for species of Pseudocalanus over species of Paracalanus.

Why did organisms for which we assume a higher energetic cost of swimming (such as Oithona spp. or organisms such as appendicularians that carry their houses) perform a significant reverse DVM when there was strong stratification, as in May 1988? Are the reductions in metabolic costs due to the lower temperature below the thermocline important enough to justify such migration (McLaren 1974)? Why are C. helgolandicus and Calanus finmarchicus not vertically segregated in the Irish Sea, as they seem to be in the Celtic Sea (Williams 1985)?

In answer to our original questions, the results of our study confirm that DVM is a very flexible behaviour that can be adopted by many planktonic organisms, depending on predation risk. However, it is not necessarily the main or only mechanism to reduce predation mortality, and predation risk seems to vary greatly between different groups or species. Some species susceptible to predation by visual predators display no DVM or a less extensive DVM than others because they use other escape mechanisms or because of energetic considerations (Bollens et al. 1993). It could also be hypothesised that the absence of DVM is a response to a relatively constant mortality risk or to a constant mortality risk/energy gain ratio situation, as has been suggested for fixed DVM (Neill 1992). Therefore, a large proportion of the planktonic community could be performing little or no DVM, while a smaller number of species perform extensive DVM, as seems to be the case in the Irish Sea.

Acknowledgements. We thank the officers and crews aboard the research vessels RRS 'Frederick Russell', RRS 'Challenger' and FRV 'Cirolana' during the series of cruises, participants on the cruises who helped in sample collection and N. R. Collins who helped in sample analysis and data processing. We also wish to thank the reviewers, whose comments contributed significantly to improving this paper. This study was supported in part by the UK Department for Environment Food and Rural Affairs (DEFRA; formerly Ministry of Agriculture Fisheries and Food) under contract GCA 10. The initial data re-analysis was stimulated by the NERC Marine Productivity Thematic Programme. X.I. is supported by a Ramon y Cajal grant from the Spanish Ministry of Science and Technology and by the Department of Fisheries and Agriculture of the Basque Country government.

\section{LITERATURE CITED}

Aksnes DL, Utne AC (1997) A revised model of visual range in fish. Sarsia 82:137-147

Alcaraz M, Strickler JR (1988) Locomotion in copepods: pattern of movements and energetics of Cyclops. Hydrobiologia 167/168:409-414

Andersen V, Gubanova A, Nival P, Ruellet T (2001) Zooplankton community during the transition from spring bloom to oligotrophy in the open NW Mediterranean and effects of wind events. 2. Vertical distributions and migrations. J Plankton Res 23:243-261

Ashjian CJ, Smith SL, Flagg CN, Wilson C (1998) Patterns and occurrence of diel vertical migration of zooplankton biomass in the Mid-Atlantic Bight described by an acoustic Doppler current profiler. Cont Shelf Res 18:831-858

Bollens SM, Osgood K, Frost BW, Watts SD (1993) Vertical distribution and susceptibilities to vertebrate predation of the marine copepods Metridia lucens and Calanus pacificus. Limnol Oceanogr 38:1827-1837

Calbet A, Alcaraz M (1996) Effects of constant and fluctuating food supply on egg production rates of Acarti grani (Copepoda: Calanoida). Mar Ecol Prog Ser 140:33-39

Choe N, Deibel D (2000) Seasonal vertical distribution and population dynamics of the chaetognath Parasagitta elegans in the water column and hyperbenthic zone of Conception Bay, Newfoudland. Mar Biol 137:847-856

Conway DVP (1980) The food of larval blue whiting, Micromesistius poutassou (Risso), in the Rockall area. J Fish Biol 16:709-723

Conway DVP, Coombs SH, Smith C (1997) Vertical distribution of fish eggs and larvae in the Irish Sea and southern North Sea. ICES J Mar Sci 54:136-147

Conway DVP, Coombs SH, Smith C (1998) Feeding success of anchovy (Engraulis encrasicolus) larvae in the north-western Adriatic Sea in response to changing hydrobiological conditions. Mar Ecol Prog Ser 175:35-49

Dale T, Kaartvedt S (2000) Diel patterns in stage-specific vertical migration of Calanus finmarchicus in habitats with midnight sun. ICES J Mar Sci 57:1800-1818

De Robertis A (2002) Size-dependent visual predation risk and the timing of vertical migration: an optimization model. Limnol Oceanogr 47:925-933

De Robertis AD, Jaffe JS, Ohman MD (2000) Size-dependent visual predation risk and the timing of vertical migration in zooplankton. Limnol Oceanogr 45:1838-1844

Duro A, Saiz E (2000) Distribution and trophic ecology of chaetognaths in the Western Mediterranean in relation to an inshore-offshore gradient. J Plankton Res 22:339-361

Falkenhaugh T, Tande K, Semenova T (1997) Diel, seasonal and ontogenetic variations in the vertical distribution of four marine copepods. Mar Ecol Prog Ser 149:105-119

Fiksen Ø, Giske J (1995) Vertical distribution and population dynamics of copepods by dynamic optimization. ICES J Mar Sci 52:483-503

Hardy AC, Gunther ER (1935) The plankton of the South Georgia whaling ground and adjacent waters, 1926-1927. Discov Rep 11:1-456

Hays GC, Proctor CA, John AWG, Warner AJ (1994) Interspecific differences in the diel vertical migration of marine 
copepods: the implications of size, colour, and morphology. Limnol Oceanogr 39:1621-1629

Hays GC, Warner AJ, Proctor CA (1995) Spatio-temporal patterns in the diel vertical migration of the copepod Metridia lucens in the northeast Atlantic derived from the Continuous Plankton Recorder survey. Limnol Oceanogr 40: 469-475

Hays GC, Warner AJ, Tranter P (1997) Why do the two most abundant copepods in the North Atlantic differ so markedly in their diel vertical migration behaviour? J Sea Res 38:85-92

Hays GC, Kennedy H, Frost BW (2001) Individual variability in diel vertical migration of a marine copepod: why some individuals remain at depth when others migrate. Limnol Oceanogr 46:2050-2054

Huntley M, Brooks ER (1982) Effects of age and food availability on diel vertical migration of Calanus pacificus. Mar Biol 71:23-31

Iwasa Y (1982) Vertical migration of zooplankton: a game between predator and prey. Am Nat 120:171-180

Lampert W (1993) Ultimate causes of diel vertical migration of zooplankton: new evidence for the predator-avoidance hypothesis. Ergeb Limnol 39:79-88

Longhurst AR (1985) Relationship between diversity and the vertical structure of the upper ocean. Deep-Sea Res 32: $1535-1570$

Lopez MDG (1996) Effect of starvation on development and survivorship of naupliar Calanus pacificus (Brodsky). J Exp Mar Biol Ecol 203:133-146

Marshall SM, Orr AP (1955) The biology of a marine copepod Calanus finmarchicus (Gunnerus). Oliver \& Boyd, Edinburgh

McLaren IA (1974) Demographic strategy of vertical migration by a marine copepod. Am Nat 108:91-102

Moraitou-Apostolopoulou M (1971) Vertical distribution, diurnal and seasonal migration of copepods in Salonic Bay, Greece. Mar Biol 9:92-98

Neill WE (1992) Population variation in the ontogeny of predator-induced vertical migration of copepods. Nature 356:54-57

Neverman D, Wurtsbaugh WA (1994) The thermoregulatory function of diel vertical migration for a juvenile fish, Cottus extensus. Oecologia 98:247-256

Nicholls AG (1933) On the biology of Calanus finmarchicus. III. Vertical distribution and diurnal migration in the Clyde sea area. J Mar Biol Assoc UK 19:139-164

Ohman MD (1986) Predator limited population growth of the

Editorial responsibility: Otto Kinne (Editor),

Oldendorf/Luhe, Germany copepod Pseudocalanus sp. J Plankton Res 8:673-713

Ohman MD (1990) The demographic benefits of diel vertical migration by zooplankton. Ecol Monogr 60:257-281

Ohman MD, Runge JA, Durbin EG, Field DB, Niehoff B (2002) On birth and death in the sea. Hydrobiologia 480:55-68

Osgood KE, Frost BW (1994) Ontogenic diel vertical migration behaviors of the marine planktonic copepods Calanus pacificus and Metridia lucens. Mar Ecol Prog Ser 104:13-25

Paffenhöfer GA, Mazzocchi MG (2002) On some aspects of the behaviour of Oithona plumifera (Copepoda: Cyclopoida). J Plankton Res 24:129-135

Pearre S Jr (1973) Vertical migration and feeding in Sagitta elegans Verrill. Ecology 54:300-314

Pearre S Jr (1980) Feeding by Chaetognatha: the relation of prey size to predator size in several species. Mar Ecol Prog Ser 3:125-134

Pearre S Jr (2000) Long term changes in diel vertical migration behavior: more ups and downs. Mar Ecol Prog Ser 197:305-307

Rakusa-Suszczeweski S (1969) The food and feeding habitat of Chaetognatha in the seas around the British Isles. Pol Arch Hydrobiol 16:213-232

Roe HSJ (1974) Observations on the diurnal vertical migration of an oceanic animal community. Mar Biol 28:99-113

Russell FS (1930) The vertical distribution of marine macroplankton. XI. Further observations on diurnal changes. J Mar Biol Assoc UK 17:767-785

Saito H, Kiørboe T (2001) Feeding rates in the chaetognath Sagitta elegans: effects of prey size, prey swimming behaviour and small-scale turbulence. J Plankton Res 23 1385-1398

Spicer JI, Stromberg JO (2002) Diel vertical migration and the haemocyanin of krill Meganyctiphanes norvegica. Mar Ecol Prog Ser 238:153-162

Timonen AG (1997) Diel vertical migrations of Calanoides carinatus and Metridia lucens (Copepoda: Calanoida) in the northern Benguela upwelling area. Oceanology 37: $782-787$

Williams R (1985) Vertical distribution of Calanus finmarchicus and C. helgolandicus in relation to the development of the seasonal thermocline in the Celtic Sea. Mar Biol 86: 145-149

Williams R, Collins NR, Conway DVP (1983) The double LHPR system, a high speed micro- and macroplankton sampler. Deep-Sea Res 30:331-342

Worthington EB (1931) Vertical movements of freshwater macroplankton. Int Rev Gesamten Hydrobiol 25:394-436

Submitted: February 3, 2003; Accepted: October 21, 2003

Proofs received from author(s): February 5, 2004 